\section{Aleitamento materno e condições socioeconômico-culturais: fatores que levam ao desmame precoce}

\section{Breast-feeding and socioeconomic cultural status: factors that lead to early weaning}

Ana Maria de Ulhôa Escobar 1

Audrey Rie Ogawa 2

Marcel Hiratsuka 3

Milka Yuri Kawashita 4

Priscila Yoshie Teruya 5

Sandra Grisi 6

Silvia Onoda Tomikawa 7

1,6 Instituto da Criança. Hospital das Clínicas da Universidade de São Paulo. Rua Dr. Enéas de Carvalho Aguiar, 647. São Paulo, SP. Brasil. CEP: 05.403-900

2-5,7 Faculdade de Medicina da Universidade de São Paulo

\section{Abstract}

Objectives: to evaluate the breast-feeding among people seeking the highlighting the causes of early weaning relating it to social, economic and cultural factors.

Methods: a sample of 599 children and caretakers who sought the Emergency Room of the Instituto da Criança, São Paulo, from of August to December 1998 were interviewed. A questionnaire asking for age, parents profession and education level, living conditions, family income, maternal work, exclusive breast-feeding length, introduction of new food, weaning reasons and the value of human milk was used.

Results: $86,1 \%$ of the mothers nursed their children and $92 \%$ claimed to be aware of the value of breast-feeding. The average weaning age was of 3,3 months; 75,9\% of the mothers weaned their children without medical advice. The reasons stated by 38,9\% of the mothers was that their milk was "thin", or "it dried" or that the child rejected the breast. Higher education of the mother and sewage availability depicted a relationship to longer breastfeeding periods ( $p=0,016$ and $p=0,011$ respectively). There was not relationship between medical follow-up in the healthcare clinic and longer breast-feeding periods.

Conclusions: although the majority of mothers were aware of the value of maternal milk and breastfed their babies, the average length of exclusive breast-feeding is lower than the nursing length advised by the World Health Organization, and that low education levels is a factor for early weaning.

Key words Breast feeding, Weaning, Cultural factors, Socioeconomic factors

\section{Resumo}

Objetivos: avaliar o aleitamento materno, ressaltando os fatores que levaram ao desmame precoce conforme as condições socioeconômico-culturais.

Métodos: analisou-se uma amostra de $599 \mathrm{cri}$ anças e seus responsáveis que procuraram o Pronto Socorro do Instituto da Criança, São Paulo, de agosto a dezembro de 1998. Utilizou-se um questionário incluindo: idade, profissão e escolaridade dos pais, condições de moradia, renda familiar, trabalho materno, duração da amamentação exclusiva, introdução de novos alimentos, causas de desmame e importância do leite materno.

Resultados: $86,1 \%$ das mães amamentaram e $92 \%$ referiram saber a importância do leite materno. A idade média do desmame foi de 3,3 meses, sendo que 75,9\% das mães suspenderam a amamentação sem orientação médica. 38,9\% referiram que o leite era "fraco", ou "secou" ou que a criança "largou" o peito. Maior escolaridade da mãe e presença de rede de esgoto mostraram relação com maior tempo de aleitamento ( $p=0,016$ e $p=0,011$ respectivamente). Não houve associação entre acompanhamento da criança no posto de saúde e tempo de aleitamento materno.

Conclusões: embora a grande maioria das mães saiba a importância do leite materno e tenha amamentado seu filho, a duração do aleitamento materno exclusivo é menor do que o preconizado pela Organização Mundial da Saúde, sendo a baixa escolaridade um fator para o desmame precoce.

Palavras-chave Aleitamento materno, Desmame, Fatores culturais, Fatores socioeconômicos 


\section{Introdução}

Ao longo da história da humanidade o leite materno tem sido a principal fonte disponível de nutrientes dos lactentes. Entretanto, a partir do século XX e principalmente após a II Guerra Mundial, o aleitamento artificial adquiriu uma importância significativamente maior.1,2 Diversos fatores contribuíram para esse fato. A industrialização e o aperfeiçoamento das técnicas de esterilização do leite de vaca propiciaram a produção em larga escala de leites em pó. As indústrias produtoras desses leites, assessoradas por intensa e agressiva publicidade procuraram fazer com que o leite em pó fosse caracterizado como um substituto satisfatório para o leite materno devido à sua praticidade, condições adequadas de higiene e suprimento completo de todas as necessidades nutricionais do lactente, uma vez que a maioria deles reforçava o fato de serem enriquecidos com variadas vitaminas, o que os tornava até superiores ao leite materno.3-6 Além disso, a entrada da mulher no mercado de trabalho limitava a possibilidade de amamentação por seis meses .

Porém, nas últimas décadas houve uma retomada da valorização do aleitamento materno. Diversos estudos comprovaram seus benefícios. Atualmente, o leite materno é preconizado como alimento exclusivo nos primeiros meses de vida da criança pela Organização Mundial da Saúde (OMS), Fundação das Nações Unidas para a Infância (UNICEF) e Ministério da Saúde.3,7-10 Dentre as vantagens comprovadas, podemos citar o valor nutricional, 4,11 a proteção imunológica devido a presença de fatores circulantes como lactoferrina, IgA secretora, anticorpos e outros, ,4,12 o menor risco de contaminação4,12 e o fortalecimento da relação afetiva entre mãe e filho. 5 Dessa maneira, o aleitamento materno diminui a morbi-mortalidade infantil4,7-10,13,14 e favorece o pleno desenvolvimento da criança.

Portanto, mostra-se importante definir os motivos que levam ao desmame precoce, a fim de proporcionar o maior tempo possível de aleitamento às crianças. Dentre os principais fatores relacionados podemos citar: nível socioeconômico,2,15 grau de escolaridade da mãe,2,16-18 idade da mãe, 16 trabalho materno,3,18 urbanização, 2,16,19 condições de parto, 16,18,20 incentivo do cônjuge e de parentes 5,17 e intenção da mãe de amamentar.5,16 O profissional de saúde também é importante no incentivo ao aleitamento materno, apoiando e instruindo a nutriz, através do acompanhamento pré-natal cuidadoso, formação de grupos de gestantes, alojamento conjunto, durante a puericultura e na promoção de campanhas de incentivo ao aleitamento.5,11,17,21 Afinal, na medida em que se conhecem os motivos que possam contribuir com o desmame precoce, pode-se atuar melhor no sentido de prevenção desses fatores de forma mais direcionada e, portanto, mais eficaz.

Este trabalho teve como objetivo estudar a situação do aleitamento materno na população atendida no Pronto Socorro do Instituto da Criança (ICr), ressaltando os múltiplos fatores que influenciam a amamentação e, principalmente, aqueles que levam ao desmame precoce.

\section{Métodos}

Trata-se de um estudo do tipo transversal realizado nos meses de agosto a dezembro de 1998, com 599 crianças e acompanhantes no serviço do Pronto Socorro do Instituto da Criança (PSICr) do Hospital das Clínicas, hospital público da cidade de São Paulo, SP, Brasil.

O Hospital das Clínicas (HC) foi utilizado por se tratar de Hospital-Escola, devendo-se lembrar que o mesmo atende diversas patologias em seu Pronto Socorro, sendo a grande maioria composta por crianças sem patologias de base. Portanto, realizaram-se as entrevistas na porta do Pronto Socorro, com os pais de crianças que vieram espontaneamente por quaisquer necessidades. Trata-se de uma população bastante heterogênea.

Considerou-se o PSICr adequado porque o aleitamento materno é um assunto de fundamental importância e que deve ser avaliado sempre, indiscriminadamente, nos três níveis de atenção à saúde: primário, secundário ou terciário.

Foi elaborado um questionário padronizado abordando dados de identificação da criança e dos pais, as condições socioeconômico-culturais e dados sobre a amamentação e assistência médica. As perguntas formuladas permitiam respostas livres, que foram agrupadas em categorias pelos entrevistadores.

Utilizou-se primeiramente um questionário piloto, sendo entrevistados cerca de 20 acompanhantes antes da definição do questionário padrão.

A aplicação dos questionários foi feita de maneira individual, na sala de espera do Pronto Socorro, sendo a escolha dos pacientes aleatória. Após consentimento verbal, as entrevistas foram realizadas pelos autores, previamente treinados, com os acompanhantes da criança, que era na maioria das vezes a própria mãe. Foram excluídos do estudo os casos em que o acompanhante não sabia fornecer dados da criança, principalmente com relação à amamentação.

Para diminuir o problema do viés de recordação, 
primeiro perguntou-se a duração do aleitamento e em seguida a idade de introdução de outros alimentos, de forma que apenas quando as duas respostas eram semelhantes o dado poderia ser considerado válido.

$\mathrm{O}$ fato de os entrevistadores conhecerem o objetivo do estudo poderia produzir viés de mensuração, porém as perguntas mais importantes solicitavam respostas objetivas (numéricas ou sim/não), como a duração do aleitamento, condições socioeconômicas, escolaridade, acesso à serviços de saúde e se o leite materno é importante. Respostas não objetivas, como qual o motivo do desmame, qual a importância do leite materno e de onde eram obtidas tais informações, não foram analisadas estatisticamente como fatores de risco para o desmame precoce.

A codificação dos questionários e a digitalização dos dados foi feita pelos entrevistadores. A análise dos dados foi feita buscando relações entre o desmame precoce (tempo de aleitamento exclusivo menor que quatro meses) e as variáveis obtidas.

Para a análise estatística ${ }^{22}$, utilizou-se o teste Qui-quadrado, adotadando-se um nível de significância de 5\%.

\section{Resultados}

A idade (Tabela 1) da maioria das mães encontrava-se nos intervalos de 21-29 anos (43,9\%) e 30-39 anos $(35,2 \%)$. Uma parcela importante $(12,3 \%)$ tinha menos de 21 anos. A idade da maioria dos pais encontrava-se nos intervalos de 21-29 anos (36,7\%) e 30-39 anos $(43,4 \%)$.
A idade média das crianças envolvidas no estudo foi 3,24 anos

A maioria das mães eram donas de casa (46\%) ou estavam empregadas (40\%) (Figura 1). Uma parcela de $13 \%$ encontrava-se desempregada e, no momento do estudo, passava o dia em casa com os filhos.

Figura 1

Porcentagem de mães empregadas.

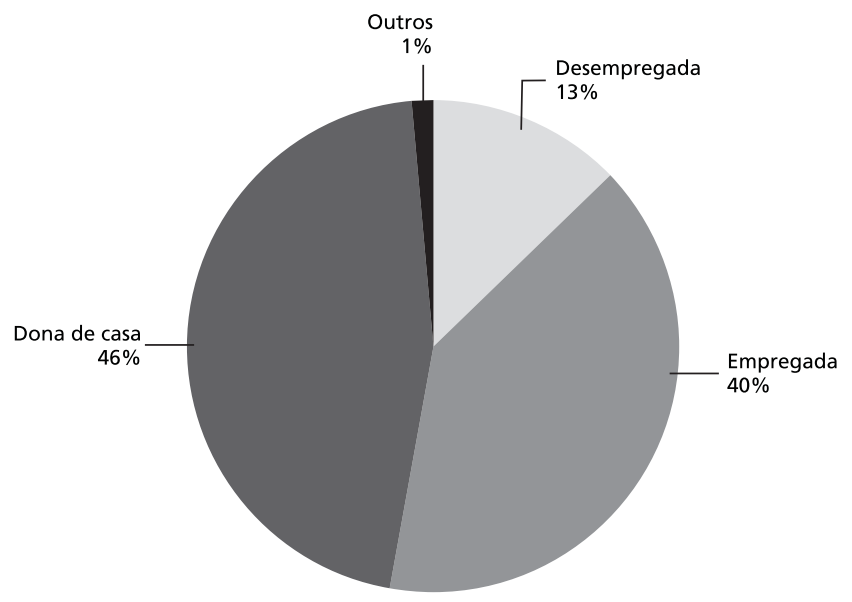

Tabela 1

Idade da mãe e do pai na população estudada.

\begin{tabular}{rrrrr}
\hline \multirow{2}{*}{ Idade } & \multicolumn{2}{c}{ Mãe $(\mathrm{N}=599)$} & \multicolumn{2}{c}{ Pai $(\mathrm{N}=567)$} \\
\cline { 2 - 5 } & $\mathrm{n}$ & $\%$ & $\mathrm{n}$ & $\%$ \\
\hline$<21$ anos & 74 & 12,3 & 15 & 2,6 \\
$21-29$ anos & 263 & 43,9 & 208 & 36,7 \\
$30-39$ anos & 211 & 35,2 & 246 & 43,4 \\
$40-49$ anos & 45 & 7,5 & 77 & 13,6 \\
$50-59$ anos & 6 & 1,0 & 17 & 2,9 \\
$>$ 60anos & 0 & 0,0 & 4 & 0,7 \\
\hline
\end{tabular}


Tempo de amamentação e variáveis analisadas (trabalho materno, escolaridade materna, presença de esgoto, seguimento no posto de saúde, pré-natal, orientação médica para suspensão, importância do leite materno).

\begin{tabular}{|c|c|c|c|c|c|c|}
\hline & \multirow{2}{*}{ Resposta } & \multicolumn{2}{|c|}{$<4$ meses } & \multicolumn{2}{|c|}{$>4$ meses } & \multirow[b]{2}{*}{$p^{*}$} \\
\hline & & $\mathrm{n}$ & $\%$ & $\mathrm{n}$ & $\%$ & \\
\hline Mãe trabalha fora? & Não & 247 & 68,8 & 112 & 31,2 & \\
\hline \multirow[t]{3}{*}{$(N=599)$} & Sim & 145 & 60,5 & 95 & 39,5 & 0,043 \\
\hline & Analfabeto & 12 & 83,3 & 2 & 16,7 & \\
\hline & Primeiro grau incompleto & 219 & 72,1 & 85 & 27,9 & \\
\hline Escolaridade da mãe & Primeiro grau completo & 60 & 59,2 & 41 & 40,8 & 0,016 \\
\hline \multirow[t]{3}{*}{$(N=598)$} & Segundo grau incompleto & 37 & 60,7 & 24 & 39,3 & \\
\hline & Segundo grau completo & 53 & 55,9 & 41 & 44,1 & \\
\hline & Superior & 14 & 58,3 & 10 & 41,7 & \\
\hline Presença de esgoto & Não & 41 & 82,0 & 9 & 18,0 & \\
\hline$(N=599)$ & Sim & 352 & 64,1 & 197 & 35,9 & 0,011 \\
\hline Segue no posto? & Não & 157 & 67,7 & 75 & 32,3 & \\
\hline \multirow[t]{2}{*}{$(N=597)$} & Regular & 38 & 65,5 & 20 & 34,5 & 0,674 \\
\hline & Irregular & 196 & 64,0 & 111 & 36,0 & \\
\hline Fez pré-natal? & Não & 10 & 56,3 & 8 & 43,7 & 0,419 \\
\hline$(N=575)$ & Sim & 368 & 66,0 & 189 & 34,0 & \\
\hline Orientação médica & Não & 311 & 68,6 & 142 & 31,4 & 0,040 \\
\hline$(\mathrm{N}=566)$ & Sim & 66 & 58,4 & 47 & 41,6 & \\
\hline Leite é importante? & Não & 40 & 84,8 & 7 & 15,2 & 0,004 \\
\hline$(N=598)$ & Sim & 353 & 64,0 & 198 & 36,0 & \\
\hline
\end{tabular}

* Teste $\chi^{2}$

Na análise estatística há dependência entre várias variáveis e o tempo de amamentação (Tabela 2). Foi observado que $31,2 \%$ das crianças mamaram mais de quatro meses no grupo em que as mães não trabalham e $39,5 \%$ no grupo em que as mães trabalham ( $p<0,043$ ), ou seja, a porcentagem de crianças que mamaram mais de quatro meses é maior no grupo em que as mães trabalham.
Devido a esse resultado aparentemente contraditório, foi verificado se as mães que trabalham fora de casa também apresentam maior escolaridade. Obtiveram-se evidências de dependência entre as variáveis $(\mathrm{p}=0,003)$, ou seja, pode-se atribuir o maior tempo de aleitamento ao fator escolaridade da mãe. 
A escolaridade materna é descrita na Tabela 3. Observou-se que apesar de ser pequena a porcentagem de analfabetas $(2,3 \%)$, a grande maioria das mães não completou o primeiro grau $(50,8 \%)$.

Observa-se na Tabela 2 que há dependência entre as variáveis $(p=0,016)$, ou seja, quanto maior a escolaridade materna, maior o tempo de aleitamento.

Em relação à profissão do pai, verificou-se que 9,4\% estavam desempregados, $88,3 \%$ estavam empregados e $2,3 \%$ enquadravam-se em outras categorias (por exemplo, aposentados).

Verificou-se também, (Tabela 3), que 4,2 \% dos pais eram analfabetos, $50 \%$ tinham o primeiro grau incompleto, $17,3 \%$ o primeiro grau completo, $8,3 \%$ o segundo grau completo, $15,2 \%$ o segundo grau completo e $4,9 \%$ o nível superior.

A renda familiar média foi de 5,16 salários mínimos.

Em 76,3\% dos casos pai e mãe moravam juntos. Encontrou-se uma média de dois filhos por casal. Em cada casa observou-se uma média de quatro habitantes. 95,5\% das casas era de alvenaria, sendo que $9,8 \%$ da população morava em favelas e $2,8 \%$ em cortiços. Nestas habitações, $97,3 \%$ tinha acesso à água encanada da rede pública e $91,7 \%$ da população estudada morava em casas com esgoto.

Houve evidência de dependência entre presença de rede de esgoto em um maior tempo de aleitamento $(\mathrm{p}=0,011)$ (Tabela 2). Observou-se que $35,9 \%$ das crianças do grupo que dispunha de esgoto mamaram mais de quatro meses, enquanto o mesmo só ocorre com 18\% das do grupo sem esgoto.

Verificou-se que $22,4 \%$ das crianças envolvidas no estudo são regularmente acompanhadas no Instituto da Criança e que $56,4 \%$ procuraram outro serviço médico antes de chegar ao Pronto Socorro.

A Figura 2 mostra o acompanhamento de saúde de rotina das crianças. Embora a maioria das crianças fosse acompanhadas regularmente nos postos de saúde $(52 \%)$, uma grande parte não tinha acompanhamento (39\%).
Tabela 3

Escolaridade da mãe e do pai

\begin{tabular}{rrrrr}
\hline & \multicolumn{2}{c}{ Mãe $(\mathrm{N}=598)$} & \multicolumn{2}{c}{ Pai (N=544) } \\
\cline { 2 - 5 } Escolaridade & $\mathrm{n}$ & $\%$ & $\mathrm{n}$ & $\%$ \\
\hline Analfabeto & 14 & 2,3 & 23 & 4,2 \\
$<$ Primeiro grau & 304 & 50,8 & 272 & 50,0 \\
Primeiro grau & 101 & 16,9 & 94 & 17,3 \\
$<$ Segundo grau & 61 & 10,2 & 45 & 8,3 \\
Segundo grau & 94 & 15,7 & 83 & 15,2 \\
Superior & 24 & 4,0 & 27 & 4,9 \\
\hline
\end{tabular}

Figura 2

Acompanhamento de saúde de rotina das crianças

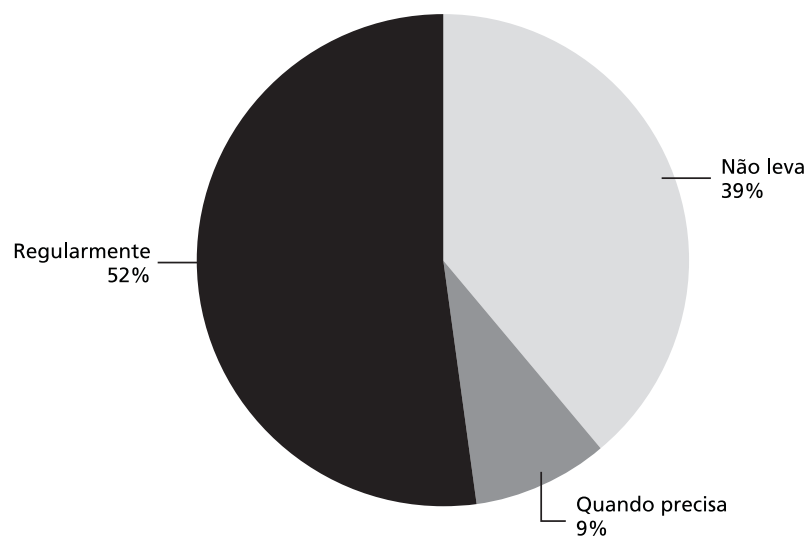


Pode-se verificou-se, ainda na Tabela 2, a não dependência entre o acompanhamento regular da criança no posto de saúde e o tempo de aleitamento.

Observou-se que $96,9 \%$ das mães envolvidas no estudo realizaram pré-natal durante a gestação. No entanto, não se verificou relação entre as variáveis, ou seja, a realização de pré-natal durante a gestação não alterou o tempo de aleitamento (Tabela 2).

Detectou-se também que $86,1 \%$ das crianças foram amamentadas pelas mães. A idade média do desmame foi de 3,3 meses de idade. A Tabela 4 apresenta a duração do aleitamento materno exclusivo e a prevalência do desmame precoce, ou seja, antes de quatro meses de idade. A Figura 3 indica as causas referidas pelas mães que as levaram a desmamar as crianças.

\section{Tabela 4}

Duração da amamentação

\begin{tabular}{crr}
\hline \multirow{2}{*}{ Duração } & \multicolumn{2}{c}{ Porcentagem } \\
\cline { 2 - 3 } & $\mathrm{n}$ & $\%$ \\
\hline < 4 meses & 290 & 56,6 \\
$4-6$ meses & 153 & 30,0 \\
$>6$ meses & 36 & 7,0 \\
Ainda mama & 33 & 6,4 \\
\end{tabular}

$\mathrm{n}=512$

Observou-se que 17,8\% das mães responderam que "o leite era fraco" ou "não sustentava", 14,7\% que "o leite secou" e $6,4 \%$ que a criança "largou o peito", totalizando $38,9 \%$ de motivos subjetivos. Em $16,9 \%$ dos casos as mães alegaram que houve orientação médica para suspensão do aleitamento materno.

Houve evidências de dependência entre as variáveis $(\mathrm{p}=0,040)$, ou seja, a orientação médica para suspensão influenciou o tempo de aleitamento materno (Tabela 2).

Em relação à importância do leite materno, 92\% das mães entrevistadas referiram conhecer a sua importância. A maioria (48\%) dos entrevistados citou como importante a defesa contra infecções, $16 \%$ citaram o valor nutricional e $19 \%$ citaram ambos os fatores (Figura 4).

A Tabela 2 mostra uma associação entre as variáveis $(p=0,004)$, indicando que conhecer a importância do leite materno influenciou o tempo de amamentação.

Finalmente 13,2\% obtiveram informações sobre a importância do leite materno pelos meios de comunicação, $32,1 \%$ através de serviços de saúde ou prénatal, e 30,6\% de ambos (Figura 5). 
Importância do leite materno referido pelas mães.

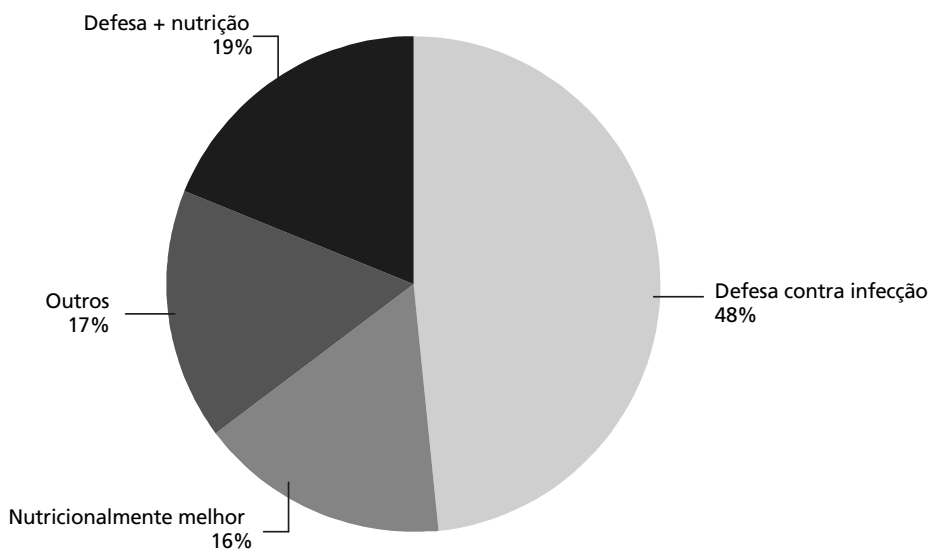

Figura 5

Modo como a mãe tomou consciência da importância do leite materno.

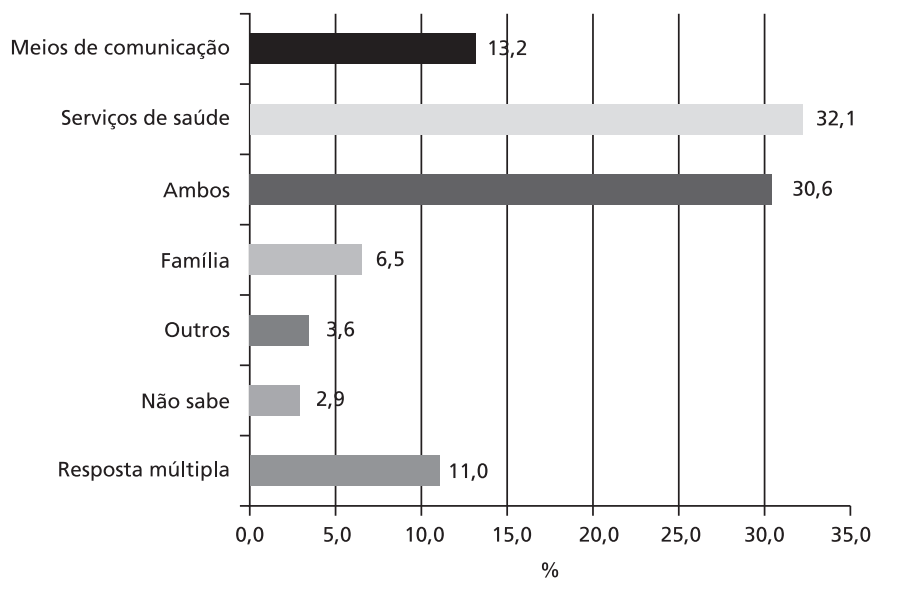

\section{Discussão}

Este trabalho avaliou uma amostra da população atendida no Pronto Socorro do Instituto da Criança. O PSICr é um centro de referência, recebendo pacientes de todo Estado de São Paulo e de outras regiões do país.

Na população estudada, $86 \%$ das mães amamentaram seus filhos. Esse resultado está abaixo do obtido em outros estudos, 18,20,23 nos quais a quantidade de mães que iniciaram o aleitamento materno foi superior a $90 \%$.

A duração média do aleitamento materno exclusivo foi 3,3 meses, portanto bem menor do que o mínimo de quatro a seis meses preconizado pela OMS. Houve dificuldades para comparar esse resultado com outros trabalhos, pois a maioria analisou a duração de aleitamento materno total, não a do exclusivo. 2,15,19,20,23,24 Apesar de durações até menores terem sido obtidas, 18 trabalhos prévios demonstraram que a maioria das crianças deixava de ser amamentada aos três meses de idade. 17,19,23,24 Estes dados demonstram claramente que o tempo de aleitamento em nosso meio foi curto e que as causas que levaram esta população ao desmame devem ser melhor consideradas no sentido de aumentar ao máximo possível o período em que as crianças podem se beneficiar com o leite de peito.

A relação entre a escolaridade materna e o tempo de amamentação é um tema complexo na literatura. Embora alguns estudos não tenham evidenciado associação entre esses fatores, 2,18 a maioria demonstra que há influência. Forman 16 revisou diversos estudos que demonstraram uma associação negativa entre o tempo de escolaridade materna e a duração do aleitamento nos países em desenvolvimento. Enquanto isso, nos países desenvolvidos, mães com maior nível de escolaridade tendem a amamentar por mais tempo. 25 Porém, o trabalho de Giugliane et al. 17 em Porto Alegre, constatou que, embora habitando um país em desenvolvimento, as mães seguiam o modelo dos países desenvolvidos, ou seja, aquelas com maior nível de escolaridade amamentaram por mais tempo. Esse resultado foi compatível com o obtido neste trabalho, já que a duração do aleitamento materno foi positivamente influenciada pelo maior nível de escolaridade da mãe, (apesar de ter sido rea-lizado em um país em desenvolvimento).

As mães que trabalham fora de casa amamentaram durante maior período de tempo. A análise estatística demonstrou que isso poderia ser explicado pelo fato de que as mães que trabalham fora de casa também apresentam maior nível de escolaridade. Assim essa variável não foi considerada relevante para 
o aleitamento, resultado semelhante ao obtido em outros estudos.3,18

A renda familiar não foi uma variável estatisticamente significante, o que é compatível com os resultados obtidos em trabalhos prévios.2,15

Em relação a condições de moradia, apenas a presença ou não de esgoto apresentou associação com o tempo de amamentação. Embora seja paradoxal, pois outros fatores não foram relevantes (casa de alvenaria, cortiço, favela, água encanada), esse dado poderia ser um marcador. Em São Paulo há favelas localizadas em bairros nobres, com ligações clandestinas de luz, água e esgoto. Mas os locais sem esgoto poderiam refletir áreas mais afastadas, com mais dificuldade de acesso a serviços de saúde.

Crianças com acompanhamento regular nos postos de saúde não foram amamentadas por mais tempo do que as não acompanhadas, e a maioria das mães $(75,9 \%)$ relata ter suspendido o aleitamento sem orientação médica. Outros trabalhos também não verificaram relação do aleitamento com o hábito de realizar controles de saúde, ${ }^{3}$ ou com o incentivo do hospital onde ocorreu o parto. ${ }^{18} \mathrm{O}$ pré-natal também não revelou associação, como já demonstrado em trabalhos anteriores.3,17,26 Este é um dado importante que deve ser analisado criteriosamente pelos responsáveis por serviços de saúde. Os profissionais envolvidos com gestantes e crianças deveriam ter também um papel educativo mais decisivo no sentido de incentivar a prática do aleitamento materno.

A maioria dos entrevistados sabia da importância do leite materno $(92 \%)$. Essa variável apresentou significância estatística, demonstrando que é fundamental tornar a população informada sobre o assun- to. A maioria obteve as informações através de meios de comunicação e serviços de saúde, reforçando, assim, a necessidade de realizar campanhas e enfatizar o aleitamento nos postos de saúde e hospitais.

Os principais motivos obtidos para introdução de outros alimentos são semelhantes aos já apresentados em estudos prévios, 3,26,27 incluindo "leite secou", "leite fraco" e "criança largou o peito" com 38,9\% das respostas. Essas respostas demonstram desconhecimento e insegurança das mães sobre o aleitamento, reiterando igualmente a necessidade de campanhas informativas sobre $\mathrm{o}$ assunto.

\section{Conclusões}

Embora a grande maioria das mães conheça a importância do leite materno e tenha amamentado seu filho, a média da duração do aleitamento exclusivo observada neste estudo foi de 3,3 meses, menor do que o mínimo preconizado pela OMS. Dos fatores analisados, os que demonstraram associação com o desmame precoce foram a baixa escolaridade da mãe e a ausência de rede de esgoto. Ao contrário do esperado, o acompanhamento da criança pelo posto de saúde não influenciou o tempo de amamentação. Assim, as ações educativas no sentido de preconizar a importância do aleitamento materno deveriam ser enfatizadas com mais vigor e insistência pelos profissionais de saúde, em todos os níveis de atendimento, para todas as crianças que, por variadas razões, entram no sistema de saúde. 


\section{Referências}

1. Bresolin AMB, Issler H, Bricks LF, Lima IN. Higiene alimentar In: Bresolin AMB, Colli AS, Marcondes E, Moysés MAA, Dias MHP. Pediatria em consultório. São Paulo: Savier; 1984. p. 38-67.

2. Issler H, Lione C, Quintal V. Duração do aleitamento materno em uma área urbana de SP, Brasil. Bol Ofic Sanit Panam 1989; 106: 513-22.

3. Figueiredo LMH, Goulart EMA. Análise da eficácia do programa de incentivo ao aleitamento materno em um bairro periférico de Belo Horizonte (Brasil) 1980/1986/1992. J Pediatr [Rio de Janeiro] 1995; 71: 203-8.

4. Cunninghan AS, Jelliffe DB, Jelliffe EFP. Breast-feeding and health in the 1980s: a global epidemiologic review. J Pediatr 1991; 118: 659-66

5. Losch M, Dungy CI, Russell D, Dusdieker LB. Impact of attitudes on maternal decisions regarding infant feeding. J Pediatr 1995; 126: 507-14.

6. Novaes HMD. A puericultura em questão [dissertação mestrado]. São Paulo: Departamento de Medicina Preventiva, Faculdade de Medicina da Universidade de São Paulo; 1989.

7. UNICEF (Fundo das Nações Unidas para a Infância). Situação mundial da infância. New York: UNICEF; 1993.

8. UNICEF (Fundo das Nações Unidas para a Infância). Situação mundial da infância. New York; UNICEF; 1996.

9. Conferência Internacional sobre Atenção Primária da Saúde: cuidados primários de saúde; 1978 6-12 set.; Alma-Ata, Casaquistão, Brasília, DF: Fundo das Nações Unidas para a Infância (UNICEF); 1978.

10. Reunião conjunta OMS/UNICEF sobre alimentação de lactentes e crianças na primeira infância: declaração, recomendações e relação dos participantes; 1979 9-12 out.; Genebra, Suíça. Brasília, DF: Fundo das Nações Unidas para a Infância (UNICEF); 1980.

11. Barros FC, Semer TC, Tonioli Filho S, Tomasi E, Victora CG. The impact of lactation centers on breastfeeding patterns, morbidity and growth: a cohort study. Acta Paediatr Scand 1995; 84: 1221-6.

12. OMS (Organização Mundial de Saúde), UNICEF (Fundo das Nações Unidas para a Infância). Proteção, promoção e apoio ao aleitamento materno. Genebra: OMS; 1989.

13. Popkin BM, Adair L, Akin JS, Black R, Briscoe J, Fliegerr W. Breast-feeding and diarrheal morbity. Pediatrics 1990; 86: 874-82.

14. Victora CG, Smith PG, Vaughan JP, Lombardi C, Fuchs SMC, Gigante LP, Nobre LC, Teixeira AMB, Moreira LB, Barros FC. Evidence for protection by breast-feeding against infant deaths from infectious diseases in
Brazil. Lancet 1987; 8554: 319-22.

15. Candeias NMF. Educação em saúde na prevenção do risco de desmame precoce. Rev Saúde Pública 1983; 17: 71 82.

16. Forman MR. Review of research on the factors associated with choice and duration of infant feeding in less-developed countries. Pediatrics 1984; 74: 667-94.

17. Giugliani ERJ, Issler RMS, Justo EB, Seffrin CF, Hartmann RM, Carvalho NM. Risk factors for early termination of breast feeding in Brazil. Acta Paediatr Scand 1992; 81: 484-7.

18. Caldeira AP, Goulart EM. A situação do aleitamento materno em Montes Claros, Minas Gerais: estudo de uma amostra representativa. J Pediatr [Rio de Janeiro] 2000; 76: 65-72.

19. Assis AMO; Prado MS; Freitas MCS; Silva RCR; Ramos LB; Machado AD. Prática do aleitamento materno em comunidades rurais do semi-árido baiano. Rev Saúde Pública 1994; 28: 380-4

20. Verronen P. Breast feeding of low birthweight infant. Acta Paediatr Scand 1985; 74: 495-9.

21. Susin LRO, Giugliani ERJ, Kummer SC, Maciel M, Benjamin ACW, Machado DB, Barcaro M, Draghetti V. Uma estratégia simples que aumenta os conhecimentos das mães em aleitamento materno e melhora as taxas de amamentação. J. Pediatr [Rio de Janeiro] 1998; 74: 368-75.

22. Agresti A. Categorical data analysis. New York: Wiley Interscience; 1990

23. Barros FC, Victora CG, Vaughan JP. Breastfeeding and socioeconomic status in southern Brazil. Acta Paediatr Scand 1986; 75: 558-62.

24. Vieira GO, Glisser M, Araújo SPT, Sales AN. Indicadores do aleitamento materno na cidade de Feira de Santana, Bahia. J Pediatr [Rio de Janeiro] 1998; 74: 11-6.

25. Bloom K, Goldblom RB, Robinson SC, Stevens AE. Factors affecting the continuance of breast feeding. Acta Paediatr Scand 1982; Suppl 300: 9-14.

26. Barros FC, Halpern R, Victora CG, Teixeira AMBB, Beria JU. Promoção da amamentação em localidade urbana da região sul do Brasil: estudo de intervenção randomizado. Rev Saúde Pública 1994; 28: 277-83.

27. Siqueira R, Durso N, Almeida AGP, Moreira MT, Massad GB. Reflexões sobre as causas do desmame precoce observadas em dinâmicas de grupo de incentivo ao aleitamento materno. J Pediatr [Rio de Janeiro] 1994; 70:1620 .

Recebido para publicação em 22 de agosto de 2001 Versão final reapresentada em 29 agosto de 2002 Aprovado em 10 de setembro de 2002 